\title{
DEFINICIÓN Y CAUSAS DE LA INFERTILIDAD
}

\author{
INFERTILITY: CAUISES AND DEFINITIONS
}

Santiago Brugo-Olmedo, M.D. ", Claudio Chillik, M.D., Susana Kopelman, M.D.

Recibido: noviembre/2002 - Revisado: febrero/2003 - Aceptado: noviembre/2003

\section{RESUIMEN}

La infertilidad es un problema común que afecta a una de cada seis parejas. Puede ser definida como la incapacidad de completar un embarazo luego de un tiempo razonable de relaciones sexuales sin tomar medidas anticonceptivas. Las causas del incremento en la prevalencia de la infertilidad son difíciles de establecer. Este aumento podría deberse por lo menos a cuatro factores: postergación del momento en que se decide tener hijos, alteraciones en la calidad del semen debido a hábitos como el tabaquismo y el alcohol, cambios en la conducta sexual y eliminación de la mayoría de los tabúes. El estudio de la pareja infértil siempre se ha enfocado considerando diferentes factores: el ovulatorio (presente en alrededor de 20\% de las parejas), el útero-tubárico-peritoneal (se observa en $\sim 30 \%$ de las parejas), el de migración del semen (10\% de los casos) y el masculino (30\% de las parejas). Cerca de $40 \%$ de todas las parejas infértiles presentan una combinación de factores y aproximadamente el 15\% no evidencia ninguna alteración objetiva que lleve a un diagnóstico definido. Durante las últimas dos décadas se registraron tres cambios importantes en el enfoque de

* CEGYR, Centro de Estudios en Ginecología y Reproducción, Viamonte 1438, Capital Federal (1055), Argentina.

Correspondencia: Tel. (5411) 4372-8289; fax (5411) 4371-7275; e-mail: sbo@cegyr.com la infertilidad. En primer lugar, la introducción de las tecnologías de reproducción asistida ha brindado una oportunidad de estudiar los procesos reproductivos básicos. En segundo lugar, han ocurrido cambios en la sociedad tales como un aumento en la proporción de mujeres mayores de 35 años que buscan el embarazo; este hecho obedece a que la gente se casa a edades más tardías y posterga el embarazo. En tercer lugar, el desarrollo de la biología molecular y de la genética se han hecho muy importantes para el estudio, diagnóstico y evaluación de las parejas, muchas de ellas consideradas hasta ahora como "parejas infértiles sin explicación”.

Palabras clave: endocrino, genético, infertilidad, mecanismos, pérdida de embarazo, embarazo.

\section{SUIMMARY}

Infertility is a common problem affecting one out of six couples. It may be defined as the incapacity to fulfill pregnancy after reasonable time of adequate sexual intercourse without contraceptive measures.

The evidence for changes in the prevalence of infertility is difficult to establish. This increase could be due to at least four factors: delayed childbearing; alterations in semen quality due to habits such as cigarette smoking and alcohol; changes in sexual behavior; and eliminations of 
most taboos. The study of infertile couple has always been focused on different factors: ovulatory factor (present in about $20 \%$ of couples), utero-tubal peritoneal factor (present in around 30\% of couples), semen migration factor (10\% of cases), and male factor (30\% of couples). Around $40 \%$ of all infertile couples exhibit a combination of factors and about $15 \%$ of couples may not display any objective alteration leading to a definite diagnosis.

During the past two decades there have been three important changes in infertility practice. First, the introduction of assisted reproduction technologies has provided an opportunity to study basic reproductive processes. Second, societal changes have occurred such as the increase in the proportion of women over 35 years old seeking for pregnancy. This fact is due to a later age for marriage and postponement of pregnancy. Third, the development of molecular biology and genetics has become very important in the study, diagnosis, and assessment of couples, many of them considered until now as "unexplained infertile couples".

Key words: endocrine, genetic, infertility, mechanisms, pregnancy losses, pregnancy.

\section{INTRODUCCIÓN}

La infertilidad es una condición que afecta al 15$20 \%$ de las parejas en edad reproductiva. Dentro del campo de la salud reproductiva, la infertilidad implica una deficiencia que no compromete la integridad física del individuo ni amenaza su vida. Sin embargo, dicha deficiencia puede tener un impacto negativo sobre el desarrollo del individuo, produciendo frustración y debilitando la personalidad, ya que la mayoría de las parejas consideran tener hijos como un objetivo de vida. Comparado con otras especies, el ser humano es altamente ineficiente en términos de reproducción. La tasa de fertilidad por ciclo es de alrededor de $20 \%$ y la de embarazos acumulados en las parejas con fertilidad probada es aproximadamente del 90\% después de doce meses y del 94\% luego de dos años (figura 1).

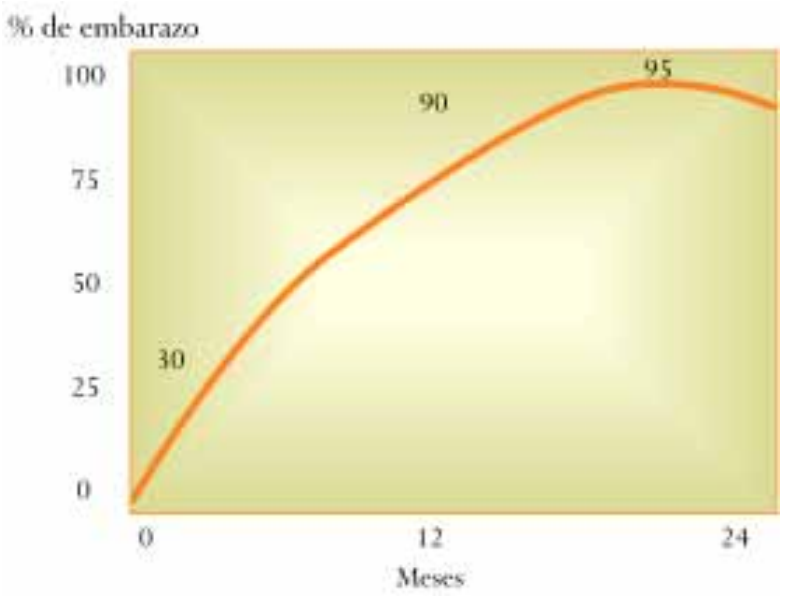

Figura 1. Tasa acumulada de embarazo en la población fértil después de dos años.

En el área de la salud reproductiva, los problemas tienden a ser diferentes en cada país. De similar manera, los estudios de poblaciones sobre este tema varían según el área estudiada. Por lo tanto, resulta de considerable interés conocer la prevalencia de la infertilidad para establecer las necesidades potenciales de la población; además, es de crucial importancia adaptar la atención sanitaria a cada población en particular.

\section{PREVALENCIA DE LA INFERTILIDAD}

\section{Definición}

La infertilidad se define como la incapacidad de completar un embarazo después de un tiempo razonable de relaciones sexuales sin medidas anticonceptivas. Los términos esterilidad e infertilidad en ocasiones son usados de manera intercambiable y algunas veces definen poblaciones diferentes. En la literatura hispana, la definición de la palabra esterilidad es la dificultad de lograr un embarazo, al tiempo que el término infertilidad es utilizado cuando se desarrolla el embarazo pero es interrumpido en algún momento; por lo tanto, es utilizado como sinónimo de pérdidas recurrentes de embarazo. Por el contrario, en la literatura inglesa el término infértil se refiere a la pareja que no logra alcanzar un embarazo, ya sea por la impo- 
sibilidad de que la mujer quede embarazada mediante los medios naturales (esterilidad), o cuando existen las posibilidades pero el embarazo no ocurre (subfertilidad), o si el embarazo efectivamente se desarrolla pero no culmina con el nacimiento de un recién nacido vivo. Por el contrario, la población fértil es definida como la de aquellas mujeres que quedan embarazadas después de un tiempo razonable de relaciones sexuales regulares.

El concepto de "tiempo razonable" es discutible; la Organización Mundial de la Salud (OMS, 1992a) así como la Sociedad Europea de Reproducción y Embriología Humana (ESHRE, 1996) en su recomendación menciona un plazo mínimo de dos años para desarrollar el embarazo; si éste no ocurre después de ese tiempo, la pareja es considerada infértil. Desde un punto de vista práctico, la mayoría de los médicos inician los estudios de una pareja infértil luego de un año de haber fracasado los intentos de embarazo. Además, debido al impacto de la edad sobre la fertilidad, cuando una mujer tiene más de 39 años, podría ser aconsejable comenzar el estudio aunque solo hayan transcurrido seis meses de intentos fracasados. En consecuencia, no se justifican los límites estrictos para comenzar un estudio de una pareja infértil, dado que el tiempo de espera debería estar relacionado con la edad de la mujer, los antecedentes de alteraciones que afectan la fertilidad, los deseos de la pareja, etc.

La fecundidad es la probabilidad que tiene la mujer de quedar embarazada en un ciclo menstrual específico y es 20\%, dependiendo de su edad. Esto lleva a que el tiempo promedio para desarrollar un embarazo sea de aproximadamente cuatro meses. La fertilidad es la capacidad de parir un recién nacido vivo.

\section{Aumento de la conciencia sobre la infertilidad y la reproducción asistida}

No hay evidencias provenientes de estudios poblacionales que sugieran una mayor incidencia de parejas infértiles; sin embargo, aparentemente en los últimos años sí ha habido un aumento del número de consultas a las clínicas de infertilidad, el cual podría ser debido por lo menos a cuatro factores.

En primer lugar, la edad promedio a la cual la mujer desea quedar embarazada ha aumentado considerablemente en las últimas décadas; su educación y participación en diferentes actividades, así como la necesidad de un avance profesional constante, la han llevado a posponer su decisión sobre el embarazo. Esto significa que las mujeres desean quedar embarazadas a una edad de aproximadamente 35 años, momento en el cual la fertilidad comienza a declinar. Así mismo, el divorcio y la búsqueda de la estabilidad con las nuevas parejas implica esperar más tiempo antes de tomar la decisión de tener hijos.

En segundo lugar, las alteraciones en la calidad del semen pueden incidir sobre la necesidad de consultar por infertilidad. Existen evidencias que muestran que hábitos como el tabaquismo (Sofikitis y col., 1995) y el abuso del alcohol son nocivos para la calidad del semen; este último, por ejemplo, ha sido relacionado con una reducción de la síntesis y secreción de testosterona y una espermatogénesis anormal. El abuso de tabaco lleva a alteraciones del espermograma.

En tercer lugar, ha habido cambios en la conducta sexual, como aumento de la frecuencia de las relaciones y del número de las parejas sexuales. Además de la decisión de retrasar el embarazo, el uso de métodos anticonceptivos (no necesariamente condones) expone a las parejas a una mayor incidencia de las enfermedades de transmisión sexual (ETS) productoras de infecciones tubárico-peritoneales, con consecuencias sobre la fertilidad.

Finalmente, la eliminación de la mayoría de los tabúes (no todos) sobre la fertilidad y la mayor difusión de los estudios existentes y de los trata- 
mientos disponibles lleva a una mayor frecuencia de consultas médicas por parte de las parejas.

\section{Edad y fertilidad}

La edad de la mujer es uno de los factores más importantes al evaluar una pareja con problemas de fertilidad. El deseo de quedar embarazada a los 40 años de edad no solo implica una baja posibilidad de éxito, sino también un aumento del riesgo de padecer dolencias maternas del embarazo como preeclampsia, hipertensión y diabetes, al igual que anomalías cromosómicas fetales y pérdidas del embarazo. La declinación de la fertilidad femenina comienza a los 30 años de edad y se hace más pronunciada a los 40 . La posibilidad de un embarazo a los 40 años de edad es del 50\% del de las mujeres más jóvenes, mientras que la incidencia de abortos espontáneos se duplica o triplica (Cruz y Gindoff, 1999) (figura 2). Según los excelentes resultados obtenidos mediante la donación de óvulos, el principal efecto que tiene la edad sobre la capacidad reproductiva de las mujeres está casi exclusivamente determinado por la edad del óvulo, dado que la posibilidad del embarazo depende más de la edad de la donante que de quien lo recibe (Sauer y col., 1990).

Fundamentalmente el efecto negativo que tiene la edad sobre el óvulo es que lo hace ineficaz para completar la primera división meiótica nor-

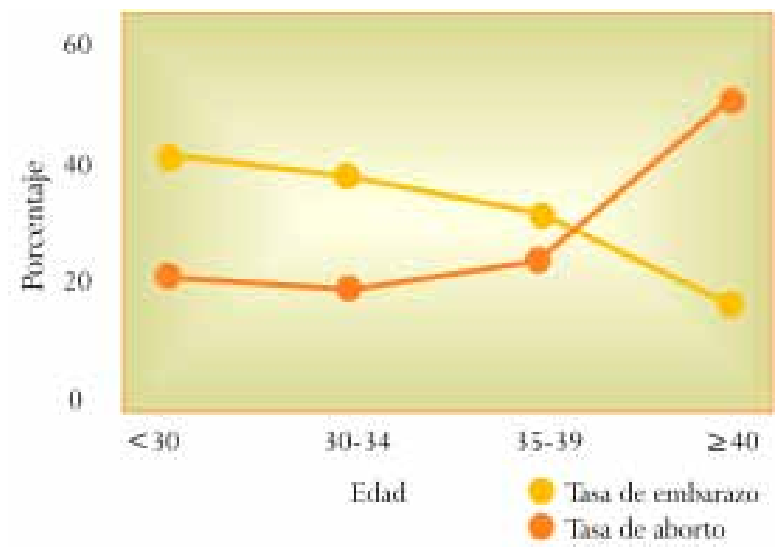

Figura 2. Tasa de embarazo y de aborto por ciclo según la edad de la mujer. mal y tal vez comenzar en forma adecuada la segunda meiosis. Por consiguiente, es defectuoso el número de cromosomas que quedan en el pronúcleo femenino al haberse completado la segunda meiosis después de la fertilización. Esto da lugar a embriones con un desequilibrio cromosómico suficientemente serio como para impedir la evolución del embarazo. La alta incidencia de trastornos durante la disyunción cromosómica del óvulo podría ser el resultado de una falla intrínseca del sistema meiótico o de un envejecimiento citoplasmático como el que resulta de una disminución de la actividad de síntesis de energía de las mitocondrias. Además de la calidad del óvulo, la edad también afecta la cantidad de folículos disponibles para la ovulación. La depleción folicular del "pool" establecido en el ovario fetal comienza en el séptimo mes de vida uterina. Por lo tanto, el número de folículos disponibles en la semana 20 de la gestación es $\sim 6-7 \times 10^{6}$ mientras que en el nacimiento es $\sim 1-2 \times 10^{6}$. Al llegar a la pubertad esta cifra disminuye a 300.000. La cantidad de ovocitos de que dispone una mujer a una edad en particular depende del equilibrio entre los ovocitos en el quinto mes de la vida intrauterina, y la proporción de ovocitos perdidos a lo largo de la vida debido a apoptosis, o como consecuencia de causas externas que pueden disminuir la reserva ovárica. Esto significa que además de la edad, otros factores afectan también la disponibilidad folicular, como agentes genéticos, anomalías cromosómicas, enfermedades autoinmunes, tabaquismo, cirugías de ovario, endometriomas, quimioterapia, radioterapia, adherencias pélvicas, exposición a Chlamydia y otros.

Muchos hombres también son subfértiles. Sobre la base de diferentes estándares para el diagnóstico clínico y seminal, la OMS (1992a) ha sugerido varios esquemas para la clasificación de la esterilidad masculina. Algunas clasificaciones se discuten hoy y muchas de ellas son descriptivas más que etiológicas. Se reconoce cada vez más la contribución de los factores ambientales, ocupacionales y especialmente genéticos. Mientras que la esterilidad es 
muy común, es bastante difícil determinar la contribución relativa del factor masculino a este problema. Muchos estudios que intentan establecer la etiología del factor masculino se basan en las investigaciones de semen propuestas por la OMS (1992b). A pesar de guardar una importancia considerable, este criterio tiene un valor diagnóstico limitado, dado que muchos hombres con parámetros seminales normales son infértiles debido al efecto de la función espermática, mientras que otros con semen teóricamente normal tienen una función espermática anormal. Hay pocos estudios epidemiológicos basados en criterios diagnósticos funcionales. Sin embargo, a pesar del uso de las técnicas diagnósticas disponibles hoy en día, el consenso actual es que el factor masculino está presente con más frecuencia de lo que se sospechaba antes en las parejas que sufren de infertilidad.

Como se sugirió previamente, el mayor obstáculo para un estudio epidemiológico significativo del factor masculino es la dificultad para realizar un diagnóstico correcto en presencia o ausencia del problema en sí. Tradicionalmente, el diagnóstico de esterilidad masculina se ha basado en el ensayo convencional del semen. Este incluye información sobre volumen, concentración espermática, motilidad y morfología. Desgraciadamente, hay un considerable número de defectos que limitan el valor diagnóstico de este estudio. El mayor obstáculo es la acentuada variabilidad intereyaculación que existe en el análisis de las muestras de semen. Además, muchos de los estudios de semen son hechos de una manera subjetiva y muestran diferencias importantes entre los distintos laboratorios e inclusive entre los técnicos de un mismo laboratorio, lo que lleva a una alta consistencia inter e intralaboratorio. A pesar de que la OMS ha establecido un rango normal de valores, éstos no se consideran en términos de su valor diagnóstico por su relación con la población fértil. Por consiguiente, en muchas parejas con un diagnóstico original de esterilidad inexplicada, al aplicarse más adelante los análisis adecuados, se puede encontrar que tienen una causa específica. Por lo tanto, parece lógico que cada laboratorio defina sus valores normales con base en sus poblaciones respectivas.

\section{Identificación de las causas de infertilidad}

Se debe realizar un estudio metódico de todos los factores probables de fracaso para alcanzar un embarazo en todas las parejas que consultan por infertilidad. El factor ovulatorio, que resume el desarrollo, maduración y la ruptura adecuadas del folículo, está presente en alrededor del 20\% de las parejas. El factor útero-tubárico-peritoneal incluye el estudio de la integridad tubárica, la cavidad uterina y la presencia de adherencias pélvicas que comprometan la anatomía del aparato genital femenino; se observa en $~ 30 \%$ de las parejas (figura 3).

El factor de migración espermática incluye el estudio de la relación entre el moco cervical y los espermatozoides. Las alteraciones en estas variables encierran una reducción en el número y la motilidad de los espermatozoides y su desplazamiento dentro del moco cervical, los cuales son prerrequisitos para llegar a las trompas y fertilizar el óvulo. Esta situación ocurre en $\sim 10 \%$ de los casos con semen normal (Cohen, 1991).

El factor masculino también corresponde al estudio del semen. Se sabe que varias afecciones provocan alteraciones en la calidad y cantidad en la muestra de espermatozoides; éstas incluyen varicocele, infecciones genitales, traumatismos, cirugías, disfunciones genéticas, sustancias tóxicas,

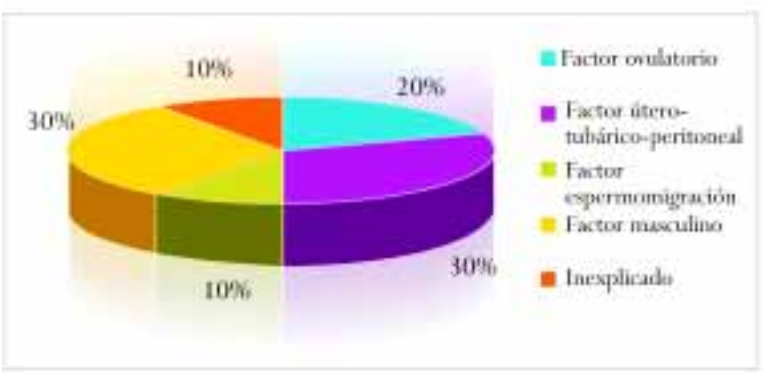

Figura 3. Distribución de las causas de infertilidad. 
etc., que ocurren en alrededor del 30\% de las parejas. La endometriosis es una patología que coexiste con infertilidad o sin ella. Si lo hace puede estar afectada la calidad de la ovulación, junto con la estructura y permeabilidad de los oviductos debido a adherencias e implantes. En opinión de algunos autores, incluso la endometriosis puede ser la causa de pérdidas de embarazo (Metzger y col.,1986).

Alrededor del $40 \%$ de todas las parejas que consultan por infertilidad habitualmente presentan una combinación de agentes; es decir, un factor femenino combinado con un trastorno masculino. Por lo tanto, el estudio de la infertilidad siempre se debe hacer considerando la pareja en conjunto: el hombre, la mujer y las interrelaciones entre ellos.

Finalmente, existen descripciones de infertilidad inmunológica e infertilidad debida a factores genéticos. Si bien estas dos categorías no corresponden a ningún tipo particular de infertilidad, la presencia de algunas alteraciones de esta naturaleza puede provocar fracaso en la reproducción.

Aproximadamente el 15\% de las parejas, inclusive cuando se han considerado todos los factores antes mencionados, pueden no exhibir ninguna alteración objetiva que lleve a un diagnóstico definitivo. Entonces son clasificados como pacientes con infertilidad inexplicada, por lo menos al momento del diagnóstico.

\section{CAUSAS DE}

\section{INFERTILIDAD FEMENINA}

\section{Infertilidad anovulatoria}

La anovulación es definida como la condición en la cual el desarrollo y la ruptura folicular están alterados y por lo tanto el ovocito no es liberado del folículo; se han identificado varias causas (Franks, 1991), las cuales encierran la insuficiencia ovárica intrínseca, que incluye factores genéticos autoinmunes, y otras como la quimioterapia. La disfunción ovárica, secundaria a la regulación gonadotrópica es otra causa. Puede subdividirse en causas específicas tales como la hiperprolactinemia y el síndrome de Kallmann, y funcionales que incluyen bajo peso corporal, exceso de ejercicio, uso de medicamentos e infertilidad idiopática. La deficiencia de gonadotropina se ve en casos de tumor hipofisario, necrosis de la hipófisis y trombosis. Pueden ocurrir alteraciones de la acción de las gonadotropinas como en el síndrome de ovario poliquístico.

En mujeres en quienes se sospecha una falla ovulatoria, las causas más frecuentes de la anovulación pueden derivar de una de las siguientes condiciones.

\section{Hiperprolactinemia}

Se pueden esperar variaciones en la dosificación de prolactina, dependiendo de las concentraciones de estrógeno en la paciente; por lo tanto, bajo condiciones hipoestrogénicas se consideran normales valores entre 20 y $25 \mathrm{ng} / \mathrm{ml}$, mientras que si la concentración estrogénica es superior, las concentraciones habituales son de 30-40 ng/ml (Lenton, 1982). La prolactina es una hormona con una sensibilidad de secreción considerable, debido a que concentraciones elevadas de prolactina pueden provenir de sustancias tales como medicaciones digestivas, antidepresivos, neurolépticos, antihipertensivos, así como condiciones de estrés, ejercicio excesivo, alta ingesta proteica, traumatismo torácico, cirugías, relaciones sexuales y otros factores. La hiperprolactinemia altera los pulsos de secreción de la hormona liberadora de gonadotropinas $(\mathrm{GnRH})$ y con base en las concentraciones de prolactina circulantes, pueden aparecer manifestaciones clínicas tales como una fase lútea inadecuada, anovulación y amenorrea. Es de rigor el estudio de la función tiroidea en todas las mujeres con hiperprolactinemia, dado que habitualmente aparece hipotiroidismo con concentraciones elevadas de prolactina (Blackwell, 1992). 


\section{Hipogonadismo hipogonadotrópico}

Esta afección se expresa por concentraciones de estradiol $<40 \mathrm{pg} / \mathrm{ml}$ y una reducción de las concentraciones de la hormona folículo-estimulante (FSH) y de la hormona luteinizante (LH). Se observa en casos con trastornos del peso y ejercicio excesivo. Puede ser idiopático o provocado por una disfunción hipofisaria o hipotalámica.

\section{Hipogonadismo hipergonadotrópico}

Las concentraciones plasmáticas de FSH pueden ser $>20 \mathrm{mUI} / \mathrm{ml}$ en determinaciones repetidas. Esta es la situación habitual en las pacientes menores de 40 años de edad con insuficiencia ovárica prematura, mujeres con ovarios resistentes o con trastornos genéticos.

\section{Ovarios poliquísticos}

Se trata de la patología endocrina de mayor prevalencia y la causa más frecuente de anovulación. Las mujeres con ovarios poliquísticos pueden presentar una amplia gama de síntomas y signos clínicos; sin embargo, la anovulación y el hiperestrogenismo son considerados prerrequisitos en esta patología. En 1844, Chereau (1844) describió cambios escleróticos en el ovario humano. En 1935, Stein y Leventhal (1935) mostraron el cuadro clásico. La elevación de LH fue descrita en 1958 (McArthur y col., 1958), pero en 1976 (Rebar y col., 1976) la afección fue definida con una LH normal. Más adelante, la presencia de este síndrome fue asociado con resistencia a la insulina y durante la década de 1980 se describieron hallazgos ecográficos en mujeres con ovarios poliquísticos. Esta cronología ilustra el amplio rango de presentaciones clínicas, la evolución de los criterios diagnósticos y otras fisiopatologías bastante oscuras.

\section{Antecedentes clínicos y científicos de la infertilidad anovulatoria}

$\mathrm{Al}$ evaluar la infertilidad ovulatoria es de fundamental importancia hacer una historia clínica cuidadosa; debe incluir la edad de la menarca, ciclos menstruales, enfermedades sistémicas (por ejemplo, trastornos de la función tiroidea), ingesta de medicamentos (tales como agentes citotóxicos, neurolépticos, antidepresivos y antihipertensivos), actividad física, situaciones de estrés y hábitos (incluyendo tabaquismo e ingesta alcohólica). El examen físico debe ser minucioso y considerar el índice de masa corporal, acné, hirsutismo, examen ginecológico que incluya el desarrollo mamario y los genitales externos e internos.

Las pruebas de detección de la ovulación pueden ser predictivas o confirmatorias; las primeras incluyen la visualización directa ecográfica del folículo dominante, los métodos indirectos tales como las concentraciones séricas de estradiol y LH y la evaluación del moco cervical. Las confirmatorias incluyen pruebas directas como ecografía o visualización laparoscópica de la ruptura folicular, y pruebas indirectas como la dosificación de progesterona, curva de temperatura basal y biopsia endometrial.

En algunas situaciones ocurre ovulación, pero la calidad de la misma es insuficiente para lograr o mantener un embarazo; tal es el caso de la fase lútea inadecuada definida por Jones (1949). Hay una transformación anormal del endometrio como consecuencia de una secreción inadecuada o insuficiente de progesterona por parte del cuerpo lúteo o por patología endometrial. Esta afección habitualmente se da con infertilidad o pérdidas recurrentes de embarazo. Según los diferentes autores, su incidencia varía de 3 a 10\% en mujeres infértiles y hasta del 35\% en pérdidas recurrentes de embarazo.

El cuerpo lúteo produce más del $80 \%$ de la progesterona circulante durante la fase lútea. Las células de la granulosa y las células de la teca presentes en el folículo que ha evidenciado ruptura forman los constituyentes de esta glándula. Dichas células toman lípidos de la circulación para sintetizar la progesterona que debería actuar so- 
bre uno de los órganos blanco, el endometrio, para alcanzar la transformación secretoria necesaria como para materializar, desarrollar y mantener el embarazo normalmente (figura 4). Cualquier alteración durante la fase folicular producirá trastornos de la fase lútea. Para el mantenimiento del cuerpo lúteo a fin de alcanzar una génesis apropiada de la fase lútea es esencial un cambio en la secreción pulsátil de LH desde una frecuencia alta y amplitud baja en la fase folicular a una frecuencia baja y amplitud alta en la fase lútea. La exposición a LH es cada vez más importante para la luteinización de las células de la granulosa y la reiniciación de la segunda división meiótica y la ruptura folicular, luteinización y mantenimiento apropiado del cuerpo lúteo. Por consiguiente, las patologías que afectan la secreción pulsátil de LH generarán defectos del nivel de luteinización adecuado de las células de la granulosa y de la génesis del cuerpo lúteo. Este es el caso en la hiperprolactinemia, estrés, desnutrición, depresión excesiva, drogas, etc.

En ausencia de suficiente sustrato para la esteroidogénesis, como en el caso de una hipobetalipoproteinemia, la síntesis de progesterona puede ser insuficiente. Se han descrito alteraciones a nivel ovárico en los receptores de las gonadotropinas, que producen anovulación o una fase lútea insufi-

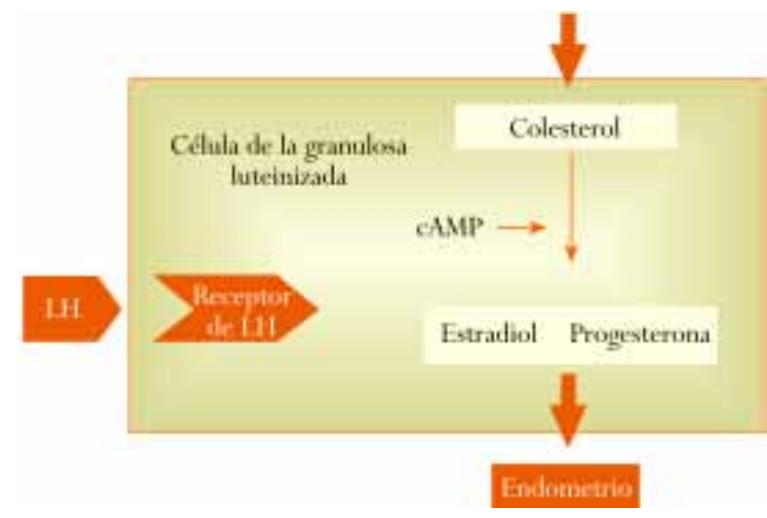

Figura 4. Representación diagramática del papel de las células luteinizadas en la regulación endocrina y en la implantación. ciente. Finalmente, compromisos endometriales como en los receptores hormonales, infecciones crónicas, etc., pueden provocar una transformación pregestacional inadecuada del endometrio.

Así como cualquier evento que reduzca la amplitud de los pulsos de LH es deletéreo, las elevaciones tónicas en la amplitud durante la fase folicular también pueden ser nocivas, en razón a una luteinización prematura de las células de la granulosa o debido a alteraciones relacionadas con el ovocito en el establecimiento precoz de la meiosis II. La luteinización celular probablemente dará lugar a una concentración elevada de la progesterona circulante en el momento del ciclo, lo cual provoca una asincronía en términos de la maduración del ovocito, la fertilización, el clivaje del embrión y la maduración endometrial. Por otra parte, al estimular la maduración a una segunda división meiótica antes de la ruptura folicular se puede generar un "óvulo viejo” incapaz de ser fertilizado (tabla 1).

Hay otras circunstancias en las cuales los factores mecánicos o biomecánicos, incluyendo la endometriosis o un pico de LH insuficiente respectivamente, completan las etapas folicular y de luteinización. Sin embargo, el folículo dominante todavía no se rompe, lo que produce la luteinización del folículo intacto y por lo tanto infertilidad.

\section{Infertilidad tubárica-peritoneal}

Los factores tubáricos-peritoneales son responsables de $\sim 30 \%$ de las causas de infertilidad. Las funciones de las trompas de Falopio están íntimamente ligadas a la integridad del epitelio ciliado responsable de la captación de ovocitos. La fertilización tiene lugar en el extremo externo de la sección ampular. Las trompas también participan en el desarrollo temprano del embrión y en su transporte a la cavidad uterina. Por consiguiente, cualquier alteración anatómica o funcional de las trompas está asociada con infertilidad. En la sociedad contemporánea, los cambios culturales que incluyen, por ejemplo, el uso de los anticoncep- 


\section{Tabla 1. Efectos deletéreos de las alteraciones de pulso de LH}

Reducción en la amplitud de los pulsos de $\mathrm{LH}$

- Luteinización inadecuada de las células de la granulosa

- Ausencia de ruptura folicular

- Luteinización y mantenimiento inapropiados del cuerpo lúteo
Elevación tónica de los pulsos de LH

- Luteinización prematura de las células de la granulosa (progesterona circulante elevada, asincronía en la maduración de los ovocitos, fertilización, clivaje del embrión y maduración endometrial

- Alteraciones de los ovocitos en el establecimiento de la meiosis II infecciones genitales provocadas por Chlamydia son actualmente la causa más importante de ETS. Este microorganismo es responsable de $\sim 60 \%$ de las salpingitis agudas en mujeres jóvenes. Se ha sugerido que las probabilidades de infertilidad por factor tubárico, así como el embarazo ectópico están considerablemente aumentadas con cada episodio infeccioso (Westrom, 1994); la tivos, han anticipado el inicio de la actividad sexual varios años antes de alcanzar una estabilidad de pareja o de inclusive considerar la fertilidad. Por lo tanto, hay un mayor riesgo de desarrollar ciertas afecciones relacionadas con la génesis del factor tubárico-peritoneal (Westrom, 1994), las cuales incluyen adherencias pélvicas secundarias a infecciones, enfermedad inflamatoria pélvica (EIP), cirugías previas o endometriosis (figura 5).

Las infecciones genitales figuran entre los principales culpables del daño tubárico-peritoneal. Muchas ETS pueden estar vinculadas indirectamente con la infertilidad, pero solo dos microorganismos han demostrado tener efectos directos sobre la fertilidad luego de la infección: Neisseria gonorrhoea y Chlamydia trachomatis (OMS, 1995). Las

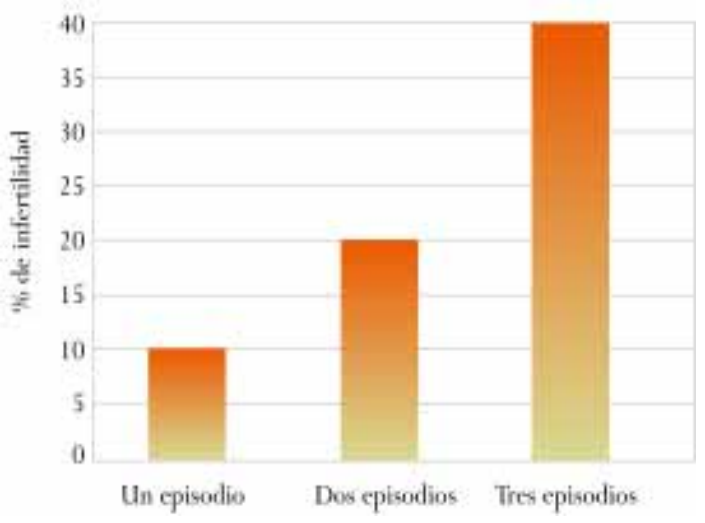

Figura 5. Número de episodios de enfermedad inflamatoria pélvica en relación con la incidencia del porcentaje de infertilidad tubárico-peritoneal. aparición de infertilidad tubárico-peritoneal también está asociada con la severidad de la infección.

En términos de prevención de las ETS asociadas con infertilidad, se deberían considerar dos líneas de acción. En primer lugar, la prevención primaria está dirigida a evitar la aparición de infecciones, y debería aconsejarse utilizar métodos anticonceptivos de barrera. En segundo lugar, la prevención secundaria requiere una evaluación y tratamiento tempranos en los casos en los que se sospecha salpingitis, con tratamiento de la pareja y control posterior a fin de evitar la reinfección.

\section{Endometriosis}

Hace mucho tiempo se estableció la asociación entre endometriosis e infertilidad. Se ha demostrado una mayor incidencia de pacientes infértiles (48\%) en comparación con individuos fértiles (5\%). Se han sugerido varias situaciones para explicar la presencia de infertilidad en pacientes con endometriosis, entre las cuales hay alteraciones anatómicas, anovulación y de la fase lútea. No obstante ello, no ha sido posible describir un único mecanismo que sea totalmente responsable de las manifestaciones clínicas de la enfermedad. Es indudable que tanto la endometriosis como las adherencias producen distorsiones anatómicas, limitan la movilización de las fimbrias y obstruyen las trompas o causan fimosis. La obstrucción tubárica distal está generalmente asociada con adherencias, mientras que las oclusiones proximales habitualmente están 
relacionadas con focos de endometriosis intramurales o con crecimiento invasor de las lesiones peritoneales (tabla 2).

Las principales opciones para evaluar la integridad tubárica-peritoneal son la histerosalpingografía y la laparoscopia. La primera es un procedimiento ambulatorio que calcula la permeabilidad tubárica y la cavidad uterina con complicaciones mínimas de infecciones y sangrado; es sumamente útil para evaluar la permeabilidad tubárica, el diámetro de las trompas y su mucosa. Sin embargo, no se puede utilizar como la única herramienta para el estudio del estado de las trompas, dado que su sensibilidad para evaluar las adherencias peritubáricas es baja. La laparoscopia realizada en la sala de operaciones bajo anestesia general permite una evaluación pélvica completa y un examen de las condiciones extraluminales y peritubáricas, así como la detección de otras afecciones, por ejemplo endometriosis. No se obtiene información sobre la luz de las trompas o el estado de la mucosa.

La histerosonografía y la sonosalpingografía pueden ser efectivas, especialmente con el uso de soluciones salinas o materiales de contraste (Holz

Tabla 2. Mecanismos propuestos de infertilidad
en pacientes con endometriosis


y col., 1997). Sin embargo, la primer evaluación morfológica del útero y de las trompas se debe realizar con histerosalpingografía; luego ir a la laparoscopia, dependiendo de cada caso particular. La histerosonografía y la salpingosonografía todavía no son sustitutos de los dos primeros métodos, pero brindan una excelente aplicabilidad y su uso puede ser bastante promisorio.

\section{Infertilidad uterina}

Hay un amplio espectro de anomalías uterinas, ya sea congénitas o adquiridas, asociadas con la presencia de infertilidad o abortos recurrentes. Entre los ejemplos se incluyen alteraciones congénitas, exposición intrauterina a medicamentos, miomas submucosos, pólipos y sinequias. Si bien efectivamente hay una asociación de ese tipo, estas afecciones también pueden darse en forma simultánea con la evolución del embarazo, lo que hace más difícil establecer una relación causa/efecto. Posiblemente esto sea debido a la falta de datos sobre la frecuencia de la aparición de estos hallazgos en pacientes infértiles. Estos tipos de alteraciones rara vez son detectados mediante el interrogatorio y el examen físico. El método de evaluación de primera línea es la histerosalpingografía.

Habitualmente la laparoscopia complementa la información en caso de alteraciones congénitas. La histeroscopia permite la evaluación y la corrección de los defectos de la cavidad uterina en el mismo procedimiento quirúrgico. La ecografía, histerosonografía y resonancia magnética (RM) contribuyen al diagnóstico de las alteraciones uterinas y brindan información sobre el aparato urinario, un blanco importante de investigación en casos de anomalías congénitas en el desarrollo del aparato genital.

\section{Migración alterada de los espermatozoides}

En 1888, Marion Sims describió la interacción entre el moco preovulatorio cervical y la motilidad espermática. El estudio de la migración espermá- tica lleva a la determinación de si es adecuada o no la relación sexual, la calidad del moco cervical y cualquier interacción entre ellos. Los constituyentes del moco son agua, electrólitos y proteínas que muestran cambios cualitativos a través del ciclo. Los estrógenos desempeñan un papel importante en la receptividad y migración de los espermatozoides dado que el moco de preovulación promueve esta actividad a un máximo. Una vez que los espermatozoides son depositados en el saco vaginal, se encuentran con el moco cervical en 180 segundos y el cérvix se convierte en un reservorio de espermatozoides que siguen moviéndose hacia arriba (primero rápido y luego lentamente) en la porción restante del aparato genital.

La manera de evaluar esta relación entre el moco y el semen en primera instancia es con la prueba postcoito o la prueba de Simms-Hubner. Es un método simple y permite la detección de alteraciones de la migración de los espermatozoides, presentes en alrededor de 10\% de las parejas que consultan por infertilidad. El resultado normal es determinado por la observación de diez espermatozoides móviles en un campo microscópico o moco periovulatorio bajo un mayor aumento. Si el resultado es malo en presencia de moco cervical adecuado y un espermograma normal, puede continuarse con una prueba in vitro de la relación moco-semen (prueba de Kremer) o se pueden utilizar pruebas cruzadas para determinar la presencia de causas masculinas o femeninas.

La presencia de anticuerpos antiespermáticos, así como ciertos agentes patógenos en el moco cervical lleva a una reducción de la motilidad espermática in vivo. Estas son causas posibles de infertilidad.

\section{Causas de infertilidad masculina}

A pesar de las dificultades diagnósticas, la OMS (1992b) sugirió un protocolo de clasificación diagnóstica para el factor masculino en las parejas 
infértiles (Rowe y col., 1993). Mediante el empleo de los datos de la OMS, Combaire y col. (1987) cotejaron informes de 33 centros en 25 países diferentes para mostrar que la incidencia de varicocele es considerable, al igual que la oligospermia idiopática (figura 6). En este estudio, la incidencia de trastornos endocrinos y genéticos fue rara. Es importante recordar que la pareja infértil presenta un gran número de factores epidemiológicos generales. Por ejemplo, la edad de la mujer ciertamente es el parámetro más importante al determinar la infertilidad. Se han detectado concentraciones espermáticas más bajas en fumadores que en no fumadores. Los avances recientes en la reproducción asistida sin duda han provocado una revolución en el tratamiento de estas parejas con factor masculino; también han contribuido a conocer mejor la etiología del factor masculino, destacando la importancia de los factores genéticos en este problema. Paradójicamente, esto ha reducido el interés en el estudio clínico del paciente, debido a las escasas posibilidades terapéuticas existentes. Entre los riesgos de adoptar un abordaje de ese tipo se incluyen las preocupaciones sobre la seguridad y los costos de las técnicas de reproducción asistida.

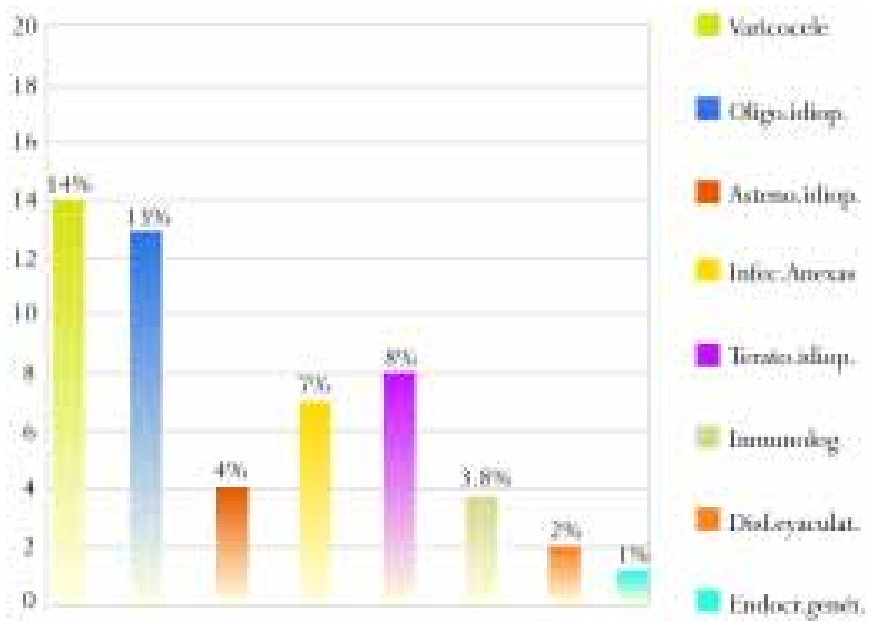

Figura 6. Distribución porcentual de las causas de infertilidad masculina.

\section{Disfunción eyaculatoria}

Existen diferentes tipos de alteraciones de la eyaculación. Aneyaculación significa ausencia de eyaculación, producida por traumatismo como en el caso de los pacientes con una sección completa o incompleta de la médula, que puede ser iatrogénica (luego de un vaciamiento ganglionar retroperitoneal), farmacológica (ingesta de antihipertensivos, antidepresivos, antipsicóticos, etc.), metabólica (diabetes) y psicológica. La eyaculación retrógrada también puede ser de origen traumático, iatrogénico, farmacológico, metabólico o psicológico.

La eyaculación prematura que impide una inseminación vaginal adecuada puede tener una causa sistémica, como en algunos casos de esclerosis múltiple. También puede ser debida a inflamaciones como las que aparecen en la prostatitis. La causa más frecuente es la psicológica.

\section{Varicocele}

El tema del varicocele ha generado controversias desde la primera publicación sobre los aparentes beneficios que surgen de tratar esta afección. Ciertamente la experiencia sugiere que el varicocele es una patología frecuente, en especial en hombres con bajas concentraciones de espermatozoides. La frecuencia observada en individuos jóvenes sanos es entre 10 y 25\%. De acuerdo con la OMS (1992), entre los hombres que consultaron al médico por problemas de fertilidad, se identificó varicocele en el 11\% de aquellos que tenían semen normal y en el 25\% con semen anormal. La mayor dificultad reside en determinar si el varicocele realmente afecta la función testicular y por ende al semen. Por otra parte, surge el interrogante de si su cura realmente mejora la fertilidad y si ese es el caso, en qué grupos de pacientes lo hace.

Aparentemente el varicocele afecta la espermatogénesis y muestra una relación clara con el semen anormal (OMS, 1992a); sin em- 
bargo, los mecanismos no han sido todavía establecidos. Ciertamente ha sido posible correlacionar una falla en la regulación de la temperatura con deterioro de la calidad de los espermatozoides en presencia de varicocele. Cualquiera que sea la fisiopatología del varicocele, hay suficiente evidencia para demostrar que produce daño testicular progresivo, y los posibles mecanismos incluyen reflujo de los catabolitos a las glándulas suprarrenales, baja concentración de oxígeno, y aumento de las concentraciones de $\mathrm{CO}_{2}$, ácido láctico y norepinefrina. Sin embargo, hay una importante discusión sobre si el tratamiento realmente mejora la fertilidad, con evidencias a favor y en contra. Inclusive hay una publicación (Sofikitis y col., 1996) que establece una capacidad reducida de los espermatozoides para desarrollar embriones con fertilización asistida en hombres con varicocele.

\section{Infección de los anexos}

Existe un segundo grupo de etiologías frecuentes que también es controvertido de acuerdo con la OMS (1995). No hay duda de que las ETS pueden producir semen patológico; sin embargo, quedan dudas sobre las infecciones subclínicas. La transmisión de enfermedades sexuales depende altamente de las razas y las culturas; en Asia, por ejemplo, hay solo una incidencia de 3\%, mientras que el valor correspondiente es de 12,2\% en África (Rowe, 1988). La gonorrea, por ejemplo, puede producir una obstrucción de los conductos seminales. La Chlamydia en los hombres puede causar una obstrucción tubárica en su pareja femenina.

Una de las consecuencias de la infección seminal es la producción más elevada de leucocitos, que puede estar asociada con un aumento de las sustancias reactivas al oxígeno. El incremento de estas sustancias está relacionado con dificultades para tener una concepción espontánea y también para la fertilización in vitro (IVF). Es importante no olvidar que los leucocitos pueden provocar una cantidad excesiva de sustancias reactivas al oxígeno a pesar de que tales aumentos también pueden ser produci- dos por los espermatozoides anormales. Por consiguiente, el diagnóstico y las implicancias terapéuticas todavía deben ser adecuadamente elucidados.

\section{Causas sistémicas y iatrogénicas}

La exposición a altas temperaturas también ha sido postulada como una causa de insuficiencia testicular, tanto en trabajadores expuestos a altas temperaturas como en aquellos que toman frecuentes baños de vapor. Igualmente se ha propuesto el efecto de la radiación en sus diferentes modalidades sobre la fertilidad, incluyendo la radiación ionizante y la electromagnética de alta frecuencia absorbida por los soldadores eléctricos, por radiólogos o por individuos que trabajan con equipos de telecomunicaciones (Weyandt y col., 1991; Larsen y col., 1991). Las personas que trabajan con plomo inorgánico, cadmio, mercurio, manganeso, cromo hexavalente, pesticidas, solventes orgánicos, gases anestésicos y monómeros plásticos o están expuestos a los mismos también corren el riesgo de desarrollar alteraciones más o menos graves de su función testicular.

De cualquier manera, queda claro que en $\sim$ $60 \%$ de los casos, independientemente de lo completo que sea el estudio del paciente, el diagnóstico etiológico en andrología aún está por determinarse.

En los últimos años, los avances en biología y genética molecular han ayudado a identificar diferentes formas de infertilidad masculina que antes eran clasificadas como idiopáticas. Estos hallazgos son extremadamente importantes porque los trastornos genéticos o de los genes que producen esterilidad pueden también afectar otros órganos no reproductores. Un caso para destacar es la agenesia bilateral de los conductos espermáticos, que es una variante leve de la fibrosis quística pancreática. Otro ejemplo es la displasia de la vaina fibrosa que puede coexistir con otras patologías del flagelo de los cilios respiratorios (Brugo y col., 1997) (tabla 3). También debe recordarse que gracias a la IVF y a la inyección intracitoplas- 
Tabla 3. Insuficiencia severa de la motilidad espermática debido a defectos del flagelo del espermatozoide

Alteraciones sistémicas

Alteraciones no sistémicas
Hipoplasia acrosómica

Síndrome de Kartagener

Displasia de la vaina fibrosa

Espermatozoides acéfalos

Ausencia de microtúbulos

del embarazo, este último depende del título de anticuerpos y la duración de la búsqueda del mismo.

El embarazo en sí podría estimular enfermedades inmunes en la mujer. La unidad feto-placentaria es un semi-injerto, ya que la contribución genética masculina tiene una expresión antigénica parcialmente desconocida para la madre. La evomática de espermatozoides (ICSI), los hombres que eran considerados como irreversiblemente estériles, en la actualidad son capaces de tener sus propios hijos. Si su esterilidad es debida a trastornos genéticos, pueden transmitir la anomalía a generaciones futuras.

\section{CATEGORÍAS ESPECIALES DE INFERTILIDAD}

\section{Infertilidad inmune}

Las respuestas inmunes entre algunos tejidos en los aparatos reproductores de la mujer y el hombre pueden ser causa de infertilidad. Está bien establecido que tanto los hombres como las mujeres pueden desarrollar anticuerpos que reaccionan contra los espermatozoides e interfieren con la fertilidad. Se cree que esto es lo que ocurre si surge aglutinación o inmovilización debido a la presencia de aquellos anticuerpos cada vez que el transporte de los espermatozoides o la fertilización están afectados (tabla 4). En los hombres pueden haber anticuerpos antiespermáticos que se adhieren a los espermatozoides en el plasma seminal y en la sangre. En las mujeres pueden aparecer anticuerpos antiespermáticos en el moco cervical, en los fluidos genitales y en la sangre. La incidencia de anticuerpos antiespermáticos es 9\% en el hombre infértil y de 13 a 15\% en la mujer infértil. Durante la evaluación de una pareja infértil, se comprueba la presencia de anticuerpos antiespermáticos si se detectan anomalías en la prueba postcoito o en el espermograma de evaluación. En relación con el pronóstico

lución normal del embarazo contradice las reglas de la inmunología del trasplante. Para cada mecanismo de protección surge una alteración que necesita ser suficientemente fuerte como para desencadenar una pérdida del embarazo. La respuesta inmune generada contra las diferencias genéticas en distintos individuos de la misma especie es llamada aloinmunidad. A fin de determinar que la pérdida del embarazo fue producida por una causa aloinmune, deben descartarse otros motivos, incluyendo los factores genéticos, infecciosos y endocrinos. La tipificación de la histocompatibilidad antígeno leucocitos (HLA) y el cultivo mixto de linfocitos son dos de las diferentes pruebas de laboratorio diseñadas para investigar tales causas.

El concepto de autoinmunidad asociado con infertilidad es un tema debatido. En las mujeres, la autoinmunidad habitualmente está relacionada con la presencia de anticuerpos contra componentes de tejido ovárico, mientras que en los hombres la autoinmunidad se refiere a los anticuerpos fundamentalmente dirigidos contra los espermatozoides. También se acepta la presencia de anticuerpos no órgano específicos; sin embargo, se discute si los anticuerpos no órgano específicos deben ser culpados de patología o si deberían ser considerados como un epifenómeno de un defecto inmune que produce infertilidad.

En las mujeres, la causa más comúnmente aceptada de autoinmunidad asociada con infertilidad es la insuficiencia ovárica prematura. La definición de esta afección es la interrupción de las menstruaciones antes de los 40 años de edad, con 
Tabla 4. Sitios de acción de los anticuerpos antiespermáticos

Transporte de espermatozoides

- Formación de grumos y aglutinación en el plasma seminal

- Inhibición de la migración a través del canal cervical

- Avance lentificado de los espermatozoides en el útero

- Destrucción de los espermatozoides por activación del complemento en el útero

Interacción de los gametos

- Inhibición de la capacitación espermática y reacción acrosómica

- Inhibición de la adherencia de los espermatozoides, unión y penetración en la zona pelúcida

- Inhibición de la fusión de los espermatozoides con la membrana vitelina del ovocito

- Inhibición de la activación de los espermatozoides y formación pronuclear

Desarrollo del embrión

- Inhibición del clivaje del ovocito fertilizado

- Acción directa contra el embrión en desarrollo para reducir la viabilidad ma folicular está agotado. La ovoforesis autoinmune puede ser uno de los extremos iniciales de la insuficiencia ovárica prematura, pero por desgracia su manifestación clínica es bastante rara. La infertilidad inexplicada y la endometriosis están asociadas con un nivel significativo de anticuerpos. El perfil inmune de estas tres afecciones habitualmente indica alteraciones de las células T.

Las pérdidas recurrentes de embarazos representan una forma de infertilidad que puede estar asociada con una función autoinmune normal. La presencia de anticuerpos no órgano específicos y órgano específicos puede predecir el riesgo de aborto. En este proceso se han implicado los anticuerpos antifosfolípidos. La insuficiencia reproductiva autoinmune, ya sea combinada con infertilidad o con pérdidas de embarazo, representa una disfunción de la célula T. El uso clínico de los anticuerpos para fines diagnósticos debería estar siempre directamente relacionado con la clínica y adaptado

el subsiguiente aumento de las gonadotropinas circulantes. La incidencia varía entre 2 y $10 \%$ en las mujeres amenorreicas y de 1 a $3 \%$ en la población en general, con anomalías autoinmunes detectadas en el $66 \%$ de los casos. De esta manera, se ha asociado la insuficiencia ovárica prematura con artritis reumatoidea, miastenia gravis, seudohipoparatiroidismo, enfermedad de Addison y alteraciones tiroideas. La insuficiencia ovárica prematura también puede ser parte de un síndrome endocrino autoinmune múltiple. No se han detectado aún antígenos específicos en humanos que desencadenen una respuesta autoinmune selectiva del ovario. Se han descrito anticuerpos circulantes, pero los métodos de detección varían en forma considerable. La investigación de los autoanticuerpos puede ser severamente obstruida porque la insuficiencia ovárica prematura puede ser la etapa final del proceso. Para el momento en que se hace el diagnóstico del efecto folicular, que es el blanco del sistema autoinmune, el siste- a cada caso en particular.

\section{Infertilidad y genética}

Los avances en la biología molecular han llevado a la detección de causas genéticas determinantes de trastornos reproductivos, tanto en hombres como en mujeres; sin embargo, esto representa un grupo muy pequeño de pacientes. Hay algunas causas con un patrón bien definido de alteraciones puramente genéticas y otras con participación de genes múltiples. Hasta el presente se han descrito formas anormales de desarrollo y de la función, que pueden ser provocadas por alteraciones a diferentes niveles del eje hipotálamo-hipófisoovárico.

\section{Genética e infertilidad femenina}

En las mujeres se pueden dividir las causas genéticas en anomalías de los cromosomas sexuales, alteraciones de los genes y otras (tabla 5). En el síndrome de Turner (45X) están implicadas 
anomalías de los cromosomas sexuales. Está afección fue descrita en 1930 en mujeres de baja estatura con disgenesia gonadal, valgo del cúbito y cuello corto. Dicho síndrome corresponde a varías anomalías de cromosomas $\mathrm{X}$ que van desde la deleción de uno de ellos (45X) a deleciones parciales asociadas con irregularidades en el ciclo menstrual y durante el embarazo (Lippe, 1991). La incidencia de diferentes tipos de variaciones del cromosoma $X$ es estimada en 1: 2.000 niños nacidos vivos. Algunas de las deleciones parciales del cromosoma X incluyen la deleción del brazo corto del cromosoma $\mathrm{X}$ que produce el síndrome de Turner típico y la deleción del brazo largo que causa una amenorrea primaria sin todos los estigmas del síndrome de Turner. Las deleciones parciales del brazo largo del cromosoma $\mathrm{X}$ originan diferentes manifestaciones clínicas, dependiendo de la región cromosómica involucrada.

Diferentes alteraciones de los genes podrían incidir en la reproducción femenina. La deleción del gen ZFX está asociada con un acortamiento de la vida reproductiva, simulando una insuficiencia ovárica temprana. El síndrome del X frágil, FMR1 está relacionado con retardo mental en los hombres, y la presencia de FRAXA (repetición del trinucleótido CGG) está asociada con insuficiencia ovárica prematura en las mujeres. Todo lo antes mencionado ocurre debido a cambios genéticos en el cromosoma X. Otras manifestaciones pueden surgir en diferentes niveles anatómicos y fisiológicos. En el gen
GnRH, el gen Kal es responsable de la migración de neuronas de la placoda olfatoria del cerebro y las modificaciones genéticas provocan defectos en el desarrollo del bulbo olfatorio (Hardelin, 1993). Este es el origen del denominado síndrome de Kallman, que incluye mutaciones del receptor para GnRH en el 7\% de las pacientes infértiles, mutaciones de los genes que codifican las gonadotropinas y sus receptores.

Las mutaciones en los genes que codifican varios factores involucrados en la síntesis de las hormonas esteroideas sexuales pueden producir in-

\section{Tabla 5. Genética de la infertilidad femenina}

\begin{tabular}{l} 
Alteraciones en el \\
cromosoma X \\
\hline Mutaciones genéticas que \\
afectan la reproducción femenina \\
ZFX \\
FRAXA \\
gen GnRH \\
Mutaciones de los genes en las \\
hormonas esteroideas sexuales \\
StAR \\
CYP 11 A \\
CYP17 \\
CYP19 \\
SDR 5 A
\end{tabular}

Mutaciones de genes en los esteroides suprarrenales

CYP 21

P.450 C21

CYP 11 B1

Mutaciones en los receptores hormonales nucleares

SF-1

DAX-1

Vida reproductiva más breve Insuficiencia ovárica prematura Síndrome de Kallman

Transporte de colesterol P.450 scc P.450 C17 Aromatasa $\alpha$-reductasa

P.450 C11

Desarrollo y diferenciación del aparato reproductor Hipoplasia suprarrenal congénita e hipogonadismo hipogonadotrópico
Receptor de estrógenos

Receptor de andrógenos 
fertilidad, por ejemplo StAR que proporciona la proteína responsable del transporte del colesterol a la membrana interna mitocondrial, citocromo P450 scc y el gen CYP 11 A, 3-B-hidroxiesteroide deshidrogenasa, citocromo P450 C17 (el gen CYP 17); B -esteroide deshidrogenasa; aromatasa (CYP 19); a reductasa (SRD 5A).

Las mutaciones genéticas que codifican para la síntesis de los esteroides suprarrenales también pueden influir en la fertilidad. Estos incluyen citocromo P450 C21 (gen CYP 21) y citocromo P450 C11 (genes CYP 11 B1). Las siguientes mutaciones génicas son efectivas en los receptores hormonales nucleares: Sf-1 que está involucrado en el desarrollo y la diferenciación del aparato reproductor, DAX-1, que es una causa de hipoplasia suprarrenal congénita e hipogonadismo hipogonadotrópico y genes de los receptores de estrógenos y de receptores de andrógenos.

Existen compromisos ginecológicos tales como el síndrome de ovario poliquístico y la insuficiencia ovárica prematura en los que podrían estar involucradas varias alteraciones genéticas. En 1985, Simpson presentó evidencias de la naturaleza genética de diferentes afecciones asociadas con infertilidad. Incluyó las anomalías müllerianas, leiomiomas, endometriosis y el síndrome de ovario poliquístico.

Es necesario hacer un estudio genético en todas las parejas que consultan por infertilidad siempre que surjan sospechas de anomalía, como en el caso de abortos recurrentes o mujeres con insuficiencia ovárica prematura.

\section{Genética e infertilidad masculina}

En términos genéticos, la infertilidad masculina puede ser clasificada como debida a un único defecto de un gen, o a un defecto numérico estructural de los cromosomas. Si el defecto afecta la producción de hormonas, el defecto involucrado es pretesticular. Si la falla del gen afecta la espermatogénesis, debería involucrarse un factor testicular. Por otra parte, cuando el defecto compromete el transporte de los espermatozoides, la causa está relacionada con un factor postesticular. $\mathrm{Si}$ el gen o el trastorno genético afectan a los espermatozoides, debería involucrarse un factor espermático.

Los defectos de un único gen que generan esterilidad masculina son debidos a mutaciones en un alelo o en dos alelos en el mismo locus. La herencia del defecto puede ser de naturaleza dominante o recesiva. La tabla 6 describe las formas más frecuentes de esterilidad masculina que surgen de un defecto de un único gen. Estos genes son considerados brevemente.

El síndrome de Kallman es un tipo de hipogonadismo hipogonadotrópico que cursa con anosmia y puede estar asociado con aplasia renal unilateral. Se trata de una afección recesiva del cromosoma X. La falla hipotalámica del agonista de GnRH puede ser debida a un defecto en el gen. Codifica para la proteína responsable de la migración neuronal desde la placa olfatoria hasta el diencéfalo, de modo que los pacientes afectados son severamente azoospérmicos u oligozoospérmicos.

La fibrosis quística pancreática es una enfermedad autosómica recesiva que resulta de un defecto de un locus con 27 exones en el brazo largo del cromosoma 7. El gen mutado es CFTR, que regula la conductancia a través de la membrana. Esta enfermedad afecta los canales del calcio y los pacientes sufren de infecciones crónicas broncopulmonares, insuficiencia pancreática y agenesia bilateral de los conductos deferentes. Sin embargo, este último defecto algunas veces es el único síntoma de la enfermedad. Como la mayoría de estos pacientes son portadores de mutaciones de CFTR, es sumamente importante estudiar a la mujer de la pareja antes de intentar la ICSI.

Desde el punto de vista genético el síndrome del cilio inmóvil es heterogéneo. El ejemplo más frecuente es el síndrome de Kartagener, caracte- 


\section{Tabla 6. Factores genéticos en la infertilidad masculina}

\begin{tabular}{|c|c|c|c|}
\hline Patología & Frecuencia & Fenotipo & Herencia/genotipo \\
\hline Síndrome de Kallman & 1 en 30.000 & $\begin{array}{l}\text { Anosmia } \\
\text { Pubertad retardada } \\
\text { Azoospermia u } \\
\text { oligozoospermia }\end{array}$ & Recesivo ligado al $\mathrm{X}$ \\
\hline Fibrosis quística & 1 en 2.500 & $\begin{array}{l}\text { Infecciones respiratorias } \\
\text { Insuficiencia pancreática } \\
\text { Desarrollo pobre del } \\
\text { tracto de Wolff } \\
\text { Azoospermia obstructiva }\end{array}$ & $\begin{array}{l}\text { Autosómico recesivo } \\
\text { Gen CFTR, cromosoma } 7\end{array}$ \\
\hline $\begin{array}{l}\text { Síndrome de } \\
\text { la cilia inmóvil }\end{array}$ & 1 en 30.000 & $\begin{array}{l}\text { Bronquiectasias } \\
\text { Situs inversus } \\
\text { Espermatozoides inmóviles }\end{array}$ & $\begin{array}{l}\text { Autosómico recesivo } \\
\text { Cromosoma } 19\end{array}$ \\
\hline Distrofia miotónica & 1 en 8.000 & $\begin{array}{l}\text { Debilidad muscular } \\
\text { Atrofia testicular }\end{array}$ & $\begin{array}{l}\text { Autosómico dominante } \\
\text { Cromosoma } 19\end{array}$ \\
\hline $\begin{array}{l}\text { Insensibilización } \\
\text { androgénica }\end{array}$ & 1 en 60.000 & Testículo feminizante & Ligado al X recesivo \\
\hline $\begin{array}{l}\text { Enfermedad } \\
\text { poliquística renal }\end{array}$ & 1 en 800 & $\begin{array}{l}\text { Riñón múltiple } \\
\text { Quistes de epidídimo } \\
\text { hígado }\end{array}$ & Autosómico dominante \\
\hline Síndrome de Usher & 1 en 30.000 & $\begin{array}{l}\text { Sordera } \\
\text { Retinitis } \\
\text { Defectos del anoxema } \\
\text { espermático }\end{array}$ & Autosómico recesivo \\
\hline
\end{tabular}

rizado por bronquiectasias, sinusitis, infecciones recurrentes del oído medio y situs inversus. La ausencia de brazos dinein en los flagelos del espermatozoide impiden la ruptura de las moléculas del ATP espermático, haciendo que el flagelo quede inmóvil. Puede ocurrir mutación en el brazo corto del cromosoma 1.

La distrofia miotónica es el resultado de una mutación de un gen en el brazo largo del cromosoma 19. Aproximadamente el $80 \%$ de los pacientes sufren de algún tipo de atrofia testicular. La insensibilidad a los andrógenos, también conocida como testículo feminizante, es una alteración en los receptores de andrógenos que obstaculiza la acción de los andrógenos circulantes. Se han descrito varias formas de la enfermedad, tales como atrofia de los músculos bulbares y espinales. La presentación más severa es la resistencia completa a los andrógenos, un trastorno recesivo del cromosoma X.

El síndrome de Usher es la causa más frecuente de sordera y ceguera hereditaria. Algunos pacientes con este síndrome presentan degeneración del axonema espermático que produce la inmovilidad total de los espermatozoides.

Hay dos tipos de anomalías cromosómicas que afectan la fertilidad masculina: numéricas y estructurales. Habitualmente hay patologías cromosómicas de novo, que resultan de mutaciones en la línea germinal parental. La anomalía cromosómica más frecuente es el síndrome de Klinefelter, 
fue la primera descrita en la literatura, y puede ser pura o en mosaico. Tiene una incidencia de 1 en 1.000 nacidos vivos y 1 en 300 fetos de abortos espontáneos. La frecuencia en los hombres estériles es $\sim 1$ a $2 \%$, pero en la población azoospérmica es $\sim 7$ a 13\%. El fenotipo prepuberal es normal, pero después de la pubertad las características más notorias son atrofia testicular bilateral con consistencia firme, deterioro de las características sexuales secundarias, algunas veces un cierto nivel de dislexia e inclusive retardo mental. El 50\% de los casos muestran ginecomastia, altas concentraciones de FSH y LH y bajas dosis de testosterona. Obviamente, casi todos estos pacientes son estériles, con oligozoospermia o azoospermia severas. Los testículos por lo regular presentan hialinosis y esclerosis tubular, pero hay algunos casos de túbulos con espermatozoides; se han descrito embarazos por fertilización asistida (Nodar y col., 1999). Hay variantes al síndrome tales como 48,XXXY, 48,XXYY y 49,XXXY. Se acepta generalmente que cuantos más cromosomas $\mathrm{X}$ presente el paciente, peor es la lesión testicular.

El síndrome de Noonan es el equivalente masculino del síndrome de Turner. Los pacientes presentan baja estatura, linfedema, orejas bajas, cuello corto, cubitus valgus y cardiopatías. La incidencia en los hombres es 1 en 2.500. La mayoría de los pacientes evidencia atrofia testicular y también es frecuente la criptorquidia. El cariotipo es 46,XO/ $\mathrm{XY}$ y la herencia es autosómica dominante. Las concentraciones de FSH están elevadas, pero las de LH y testosterona son normales. Por lo general estos pacientes son severamente azoospérmicos u oligozoospérmicos.

Los hombres con el síndrome 47,XYY son fenotípicamente sanos; este trastorno se da con una incidencia de 1 en 1.000. Quienes lo presentan pueden tener un mayor riesgo de trastornos de la personalidad e inclusive muestran comportamiento antisocial. Algunos de estos pacientes sufren de oligozoospermia, pero muchos de ellos son normo- zoospérmicos. El riesgo de tener hijos anormales es mínimo, dado que el cromosoma $\mathrm{Y}$ adicional se pierde precozmente en la línea germinal y por lo tanto no pasa a la próxima generación.

Las deleciones del cromosoma Y pueden estar asociadas con infertilidad masculina. Aproximadamente el $10 \%$ de los hombres con oligozoospermia y el 15\% de los pacientes con azoospermia genética presentan una deleción del factor de azoospermia (AZF), que está localizado en el brazo largo del cromosoma Y (Reijo y col., 1996). Hasta la fecha se han identificado varias secuencias de genes, localizados en el brazo largo del cromosoma Y, que son candidatos para el gen de azoospermia. El gen que muestra deleción más frecuente en la azoospermia es DAZ, en el 70\% de los casos. Sin embargo, este gen también ha sido secuenciado en el cromosoma 3. Codifica para una proteína con 366 aminoácidos involucrada en la regulación de la meiosis. Los pacientes portadores de este defecto que puedan convertirse en padres luego de ICSI presentan un alto riesgo de transmitirlo a su descendencia.

Las translocaciones pueden ser de naturaleza balanceada o robertsoniana. Esta última es diez veces más frecuente en hombres estériles o en aquellos con semen patológico. La translocación más frecuente es 13;14. Puede estar acompañada de semen normal o de una oligozoospermia severa, e inclusive con azoospermia.

Las alteraciones en el ADN mitocondrial recientemente han sido relacionadas con defectos del semen. Un ejemplo identificado en varios pacientes con oligozoospermia comprende la deleción del gen 4977 mtADN, responsable de esta anomalía seminal.

\section{Resumen de las enfermedades genéticas}

Es importante destacar el incremento en la descripción de las enfermedades genéticas o de los genes que explican los trastornos seminales que 
producen infertilidad. Debe recordarse este concepto cuando se administran tratamientos tales como la ICSI, debido a las futuras consecuencias hereditarias. Estas consecuencias no necesariamente son malformaciones, y la frecuencia de malformaciones en los niños recién nacidos concebidos a través de ICSI o IVF parecen ser equivalentes. Podría haber graves problemas de fertilidad para los bebés recién nacidos o, incluso peor, alteraciones en la genética de la población que representa un riesgo para las generaciones futuras.

Se puede sugerir la realización de diagnósticos genéticos preimplantación de embriones que se están clivando para mujeres mayores de 35 años de edad. El diagnóstico de aneuploidía en espermatozoides con hibridización in situ y el uso de fluorocromos (FISH) está indicado para la oligoastenoteratozoospermia severa, teratozoospermia severa, parejas con abortos recurrentes y aquellas que no tienen causa aparente de infertilidad. Luego del tratamiento se han descrito alteraciones cromosómicas de novo en gónadas y embriones. El asesoramiento genético antes, durante y después del tratamiento de reproducción asistida es una práctica sólida para hacer un diagnóstico correcto y adaptar las recomendaciones genéticas específicas a las parejas y sus familias.

\section{Infertilidad inexplicada}

La infertilidad inexplicada o sin causa aparente es un término usado para aquellos casos en los que los estudios de la infertilidad muestran resultados normales. Esta situación ocurre en alrededor del 15\% de las parejas y habitualmente es frustrante, tanto para el médico como para la pareja, debido al sentimiento de expectativas perdidas al no disponer de un diagnóstico específico. Las parejas con infertilidad inexplicada podrían tener un defecto sutil en su capacidad reproductiva, que no puede ser identificado a través de una evaluación estándar. También es posible la exis- tencia de causas específicas, pero desconocidas hasta la fecha o que su detección está más allá del dominio de los procedimientos diagnósticos disponibles (Crosignani y col., 1993).

Desde un punto de vista terapéutico puede ser importante considerar a estas parejas como individuos con una capacidad reproductiva limitada, dado que muchas alcanzan el embarazo pero les lleva más tiempo que a las parejas normales. Si se considera de esa manera, la razón para administrar un tratamiento para la infertilidad cuando no se ha identificado una causa aparente sería aumentar la probabilidad mensual de alcanzar un embarazo. En relación con el pronóstico de estas parejas, la duración de la infertilidad se convierte en una información importante. Después de tres años de infertilidad no tratada, la tasa de embarazo por año cae $24 \%$ cuando una mujer es mayor de 30 años de edad. El pronóstico es más optimista en parejas con historia de embarazos previos. Los individuos con infertilidad inexplicada deberían ser conscientes del hecho de que es probable que el embarazo ocurra sin tratamiento, pero que les llevará más tiempo que a otras parejas.

\section{CONCLUSIONES}

La infertilidad es una afección que afecta del 16 al 20\% de las parejas en edad reproductiva. Para investigar su causa es necesario estudiar a ambos individuos, dado que el $40 \%$ de todas las parejas infértiles presentan una combinación de causas. El factor ovulatorio necesita una anamnesis exhaustiva, examen físico, probable detección de la ovulación y perfil hormonal para una evaluación completa. El factor tuboperitoneal representa aproximadamente el 30\% de las causas de infertilidad. Las principales opciones para evaluar este factor son la histerosalpingografía y la laparoscopia. La endometriosis tiene alta incidencia en las pacientes infértiles (48\%) y se han propuesto varios mecanismos en 
la endometriosis relacionada con la infertilidad. El factor masculino está presente en el 30\% de las parejas y varias enfermedades están relacionadas con la infertilidad masculina. El varicocele es la patología más frecuente, seguida de oligozoospermia idiopática. El estudio de la infertilidad, tanto femenina como masculina, ha sido extensamente influido por el desarrollo de la biología molecular y la genética. Estas dos disciplinas ahora son cruciales para el estudio, diagnóstico y evaluación de las parejas infértiles.

\section{REFERENCIAS}

Blackwell R. Hyperprolactinemia: evaluation and management. In: Moghissi K (ed). Endocrinology and metabolism. Clin North Am 1992; 21: 105.

Brugo Olmedo S, Nodar F, Chillik C, Chemes H. Successful intracytoplasmic sperm injection with spermatozoa from a patient with dysplasia of the fibrous sheath and chronic respiratory disease. Hum Reprod 1997; 12: 1497-1500.

Cohen B. The postcoital test. Infertility and reproductive medicine. Clini North Am. Saunders, Philadelphia, USA; 1991. p 317.

Comhaire FH, De Kretser D, Farley TMM, et al. Towards more objectivity in diagnosis and management of male infertility: results of World Health Organization multicentre study. Int J Androl 1987; 10: 1-53.

Crosignani P, Collins J, Cooke I, et al. Unexplained infertility. Hum Reprod 1993; 8: 977.

Cruz JR, Gindoff PR. Age and reproduction. Reproductive Medicine Review 1999; 7: 61-69.

Franks S. Diagnosis and treatment of anovulation. In: Hillier SG (ed). Ovarian endocrinology. Blackwell Scientific Publications, Oxford, UK; 1991. p. 227-238.

Hardelin J. Xp22.3 deletions in isolated familial Kallman's syndrome. J Clin Endocrinol Metab 1993; 76: S27 - S91.

Holz, et al. Ultrasound in the investigation of tubal patency: a meta-analysis of three comparative studies of Echovist-200 including 1007 women. Zentralbl Gynäkol 1997; 119: 366-373.
Jones G. Some newer aspects of the management of infertility J Am Med Assoc 1949; 141: 1123.

Lenton E. The human menstrual cycle: plasma concentrations of prolactin, LH, FSH, oestradiol and progesterone in conceiving and non-conceiving women. J Reprod Fertil 1982; 65: 131-139.

Larsen AI, Olsen J, Svane O. Gender-specific reproductive outcome and exposure to highfrequency electromagnetic radiation among physiotherapist. Scand J Work Environ Health 1991;17: 324-329.

Lippe B. Turner syndrome. Endocrinology and metabolism. Clin North Am 1991; 20: 121.

Metzger D, Olive D, Stols G. Association of endometriosis and spontaneous abortion effect of control group selection. Fertil Steril 1986; 45: 18.

Moghissi K. Significance and prognostic value of the postcoital test. In: Insler V, Bettendorf (eds). The uterine cervix in reproduction. George Thiene, Stuttgart, Germany; 1977. p 231.

Nodar, F de Vincentiis, Brugo Olmedo S, Papier $\mathrm{S}$, et al. Birth of twin males with normal karyotype after intracytoplasmic sperm injection with use of testicular spermatozoids from a non-mosaic patient with Klinefelter's. Fertil Steril 1999; 71: 1149-1152.

Reijo R, Alagappan RK, Patricio PY, Page DC. Severe oligozoospermia resulting from deletions of azoospermia factor gene on Y chromosome. Lancet 1996; 347: 1290-1293.

Rowe PJ, Comhaire FH, Hargreave TB, Mellows HJ. WHO manual for the standardized investigation and diagnosis of the infertile couple. Cambridge University Press, Cambridge, UK; 1993.

Rowe PJ. WHO's approach to the management of the infertile couple. In: Negro Vilar A, Isidori A, Paulson J, et al (eds). Andrology and Human Reproduction. MPP Serono Symposia Publications, Raven Press, New York, USA; 1988. p 219-309.

Sauer M, Paulson R, Lobo R. A preliminary report on oocyte donation extending reproductive potential to women over 40. New Engl J Med 1990; 323: 1157.

Sofikitis N, Migayana I, Dimitriadis D. Effects of smoking on testicular function, semen quality and sperm fertilizing capacity. J Urol 1995; 154: 1030. 
Stein I, Leventhal M. Amenorrhoea associated with bilateral polycystic ovaries. Am J Obstet Gynecol 1935; 29: 181 .

Westrom L. STD and infertility. Sexually Transmitted Diseases 1994; 21: S32-S37.

Weyandt TB, Shrader SM, Turner TW, et al. Semen analysis of samples from military personnel associated with military duty assignments. J Androl 1991; 13: 1-29.
World Health Organization. The influence of varicocele on parameters of fertility in a large group of men presenting to infertility clinics. Fertil Steril 1992; 57: 1289-1293.

World Health Organization. Task force on the prevention and management of infertility: serologic relationship to post-chlamydial and gonococcal infection. Sexually Transmitted Diseases 1995; 21 : 71-77. 